\title{
Energy-efficient time and energy resource allocation in non-selfish symbiotic cognitive relaying sensor network with privacy preserving for smart city
}

\author{
Ping Li, Yu Liu, Xiang Gao, Huibo Li and Peng Gong* (D)
}

\author{
${ }^{*}$ Correspondence: \\ penggong@bit.edu.cn \\ School of Mechatronical \\ Engineering, Beijing \\ Institute of Technology, \\ Beijing 100081, China
}

\begin{abstract}
Energy efficiency and privacy preserving have become essential for the wireless sensor networks. In this paper, a joint energy and time resource allocation problem for the cognitive users (CUs) in a non-selfish symbiotic cognitive relaying scheme (NSCRS) is considered. We aim to maximize the total energy efficiency (EE) of the primary user and CUs with the consideration of information privacy under the total energy constraints of CUs. With full channel state information (CSI), an optimal energy and time resource allocation algorithm is proposed based on the exhaustive searching. Besides, in order to reduce the overhead of CSI feedback, a suboptimal algorithm, in which only the partial instantaneous CSI is required, is additionally proposed. Simulation results demonstrate the EE of primary and CUs in the NSCRS with consideration of information privacy can be greatly improved by the proposed algorithms.
\end{abstract}

Keywords: Wireless sensor network, Energy efficiency, Primary user, Cognitive relaying, Privacy preserving

\section{Introduction}

Recently, the wireless sensor network (WSN) with privacy preserving has been widely considered in the civilian fields [1,2]. However, due to the limited power supply for sensor nodes, energy efficiency of relay assisted WSN has attracted more and more attention $[3,4]$. In addition, owing to better spectrum efficiency via relays with cognitive function, the radio resource management for EE of cognitive relay assisted WSN with privacy preserving is valuable for researching.

Owing to a larger service coverage and a higher system capacity at a relatively low deployment cost, relays had been widely considered into WSN to prolong the lifetime of network [5-10]. There are usually two kinds of relays, amplify-and-forward (AF) relay and decoded-and-forward (DF) relay. The AF relay simply forwards the received signal to the destination, whereas the DF relay needs to decode the signals before the transmission [11]. In [12], a three-layered architecture was proposed for randomly deployed heterogeneous wireless sensor networks, where a minimum

(c) The Author(s) 2021. Open Access This article is licensed under a Creative Commons Attribution 4.0 International License, which permits use, sharing, adaptation, distribution and reproduction in any medium or format, as long as you give appropriate credit to the original author(s) and the source, provide a link to the Creative Commons licence, and indicate if changes were made. The images or other third party material in this article are included in the article's Creative Commons licence, unless indicated otherwise in a credit line to the material. If material is not included in the article's Creative Commons licence and your intended use is not permitted by statutory regulation or exceeds the permitted use, you will need to obtain permission directly from the copyright holder. To view a copy of this licence, visit http:// creativecommons.org/licenses/by/4.0/. 
energy consumption algorithm for relay node selection was presented to improve the network lifetime. The author in [13] investigated a load balancing strategy of optimal number of relays for deploying for a longer network lifetime. Meanwhile, a minimum number of relay nodes, which is utilized to enhance the outage probability, was obtained by the proposed relay deployment algorithm [14]. Besides, a novel connectivity-aware approximation algorithm for best relay node placement was proposed to offer a major step forward in saving system overhead in the wireless sensor networks [15]. And a non-orthogonal AF (NAF) scheme, where all the relays were allowed to transmit signals in the same time and frequency simultaneously, was considered and a higher spectral efficiency could be achieved compared to the orthogonal AF scheme [16-18]. However, the EE as well as transmission model with privacy preserving was not considered in [5-18].

Meanwhile, a demand-based load balancing algorithm was addressed for energy-efficiency in WSN to improve the network life-cycle and ensure the communication quality simultaneously in [19]. A cooperative privacy preserving scheme, in which an opportunistic user selection policy was investigated to optimize the secrecy performance, was proposed in multiuser relay network [20]. However, the cognitive relay function as well as the NAF relaying was not further considered in $[19,20]$. Furthermore, cognitive radio is regarded as an effective approach for enhancing the utilization of the radio electromagnetic spectrum [21]. In [22], a distributed connection restoration algorithm, in which cognitive function based relays were considered, was proposed to ensure the connection of WSN with a minimum number of relays. The authors in [23] considered a WSN, where a cognitive relay assisted the primary transmitter was assumed, and thus, the throughput for both primary and secondary systems could be maximized. By optimizing the sensing time as well as the power allocation in multi-channels, the EE of the WSN could be maximized with the assistance of multi-hops DF relay [24]. However, all the investigated schemes or algorithms in [22-24] only considered the orthogonal transmission among relays and the NAF relaying as well as the privacy preserving was not taken into account.

In this paper, we intend to maximize the overall energy efficiency by optimally allocating the energy and time among CUs, while minimizing the required interaction between primary and cognitive networks as well as the overhead of CSI feedback, in a WSN with consideration of privacy preserving, in which an access point (AP) is utilized to broadcast the artificial noise and such noise is eliminated at the destination node to protect the information privacy. We first formulate the energy and time allocation problem to maximize the energy efficiency of NSCRS [25] with privacy preserving under a sum energy constraint at CUs. Then, an optimal energy and time allocation algorithm is proposed based on the full CSI feedback. In order to reduce the overhead of CSI feedback, another optimal algorithm based on partial CSI feedback, in which only the instantaneous CSI of $\mathrm{PU}_{s}-\mathrm{PU}_{d}$, instantaneous CSI of $\mathrm{PU}_{s}-\mathrm{CU}_{s}$, and the average value of CSI between CUs and $\mathrm{PU}_{d}$ for each fading block rather than an instantaneous value are required, is proposed for the slow fading channel environment.

The remainder of this paper is organized as follows. Section 2 gives a detailed description of the system model. In Sect. 3, the optimal joint energy and time resource allocation problem for NSCRS is addressed. In Sect. 4, the proposed optimal and suboptimal 
algorithms are given in details. Intensive simulations are conducted to evaluate the proposed algorithms in Sect. 5. Finally, the paper is concluded in Sect. 6.

\section{System model}

In this section, the system model, including system architecture and transmission models for both conventional cognitive radio scheme (CCR) and NSCRS with consideration of privacy preserving, for the network with coexistence of primary and cognitive users is presented in detail.

\subsection{System architecture for coexistence of primary and cognitive networks with consideration of privacy preserving}

We consider a system that consists of primary and cognitive networks as shown in Fig. 1. We assume that the primary network is a time division multiple address (TDMA)-based half-duplex network, in which the $\mathrm{PU}_{s}$ transmits messages to different $\mathrm{PUs}$, i.e., $\mathrm{PU}_{d}$, in different time slots and nodes cannot transmit and receive simultaneously. In the cognitive network, CUs seek opportunities to access the AP of cognitive network and CUs will cooperate with $\mathrm{PU}_{s}$ when the energy efficiency is better than that of the direct transmission from $\mathrm{PU}_{s}$ to $\mathrm{PU}_{d}$. For the symbiotic architecture, CUs can send messages to AP only when CUs have incentive time obtained from the cooperative transmission to the PU. Besides, there are undesired nodes, which are viewed as potential eavesdroppers, around $\mathrm{PU}_{d}$. Therefore, to prevent privacy leakage, it is assumed that the AP broadcasts two kinds of artificial noise (AN) in phases 1 and 2, respectively, when CUs are considered as relays. While the privacy preserving is assumed to be based on the acknowledgement of CSI at AP, which can be obtained by the handshake procedure [20]. Moreover, for simplicity, we assume that the channels among $\mathrm{PU}_{s}, \mathrm{CUs}, \mathrm{AP}$, and $\mathrm{PU}_{d}$ are quasistatic, independent and identically distributed (i.i.d.), which means that the channel state will remain constant within a fading block and vary independently and identically from one fading block to another. In addition, the flat Rayleigh fading channel is assumed, that is, the fading channel will remain almost unchanged over long enough duration for channel estimation, cooperation, and data transmission. Besides, a control channel for the delivery of CSI, cooperation parameters and incentive time allocation is also considered $[25,26]$.

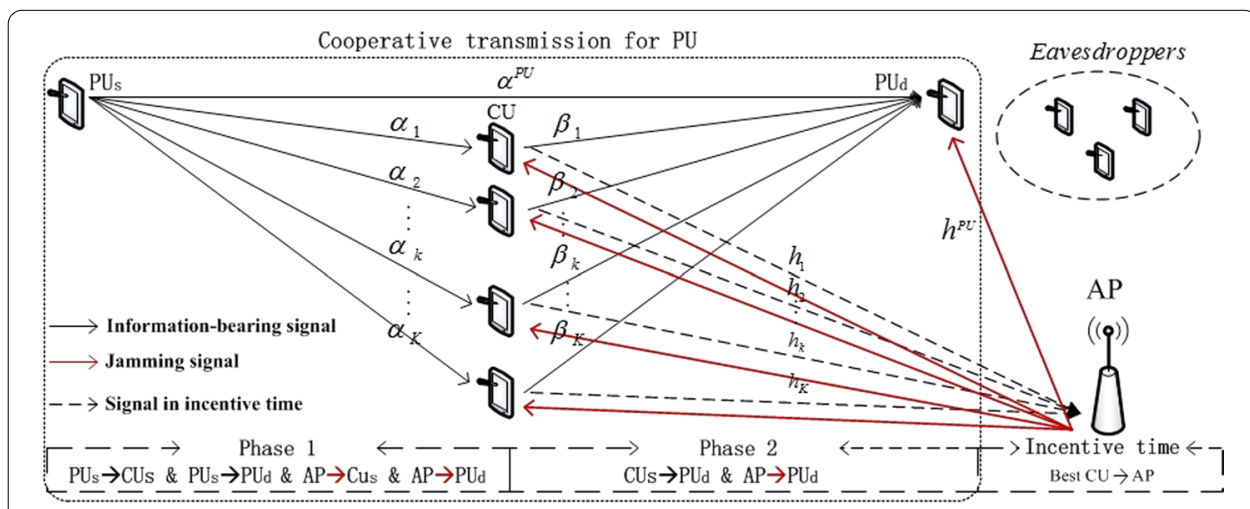

Fig. 1 System architecture for coexistence of primary and cognitive networks with privacy preserving 


\subsection{Transmission methods for CCR and NSCRS with privacy preserving}

The transmission method of CCR is shown in Fig. 2a. The $\mathrm{PU}_{s}$ has a constant power of $P_{\mathrm{PU}}$ for both CCR and NSCRS. And the transmission time is assumed to be $T$ seconds. For the privacy preserving, it is assumed that the AP will broadcast the AN, which is known at the $\mathrm{PU}_{d}$, in CCR. Therefore, the additive variable of $\mathrm{AN}$ at $\mathrm{PU}_{d}$ can be eliminated owing to the acknowledgement of AN. However, for the undesired nodes, the AN cannot be removed. Thus, the received signal power at desired PU, i.e., $\mathrm{PU}_{d}$, can be given as $P_{D}$,

$$
P_{D}=P_{\mathrm{PU}} \alpha^{\mathrm{PU}}=\frac{E_{\mathrm{PU}} \alpha^{\mathrm{PU}}}{T},
$$

where $E_{\mathrm{PU}}$ denotes the total transmission energy at PU over time of $T$ and $\alpha^{\mathrm{PU}}$ is the channel gain of $\mathrm{PU}_{s}-\mathrm{PU}_{d}$ due to fading, path loss and shadowing. In addition, the energy consumption of AP in CCR is assumed to be $E_{\mathrm{AP}}$.

And the received signal-to-noise ratio (SNR) at $\mathrm{PU}_{d}$ for CCR, $\gamma_{\mathrm{PU}}$, can be given as

$$
\gamma_{\mathrm{PU}}=\frac{P_{D}}{N}=\frac{E_{\mathrm{PU}} \alpha^{\mathrm{PU}}}{N T},
$$

where $N$ represents the power of additive white Gaussian noise (AWGN). Then, the rate of primary transmission at $\mathrm{PU}_{d}$ in $\mathrm{CCR}$ can be given as

$$
R_{\mathrm{PU}}=\log _{2}\left(1+\gamma_{\mathrm{PU}}\right)
$$

Thus, the number of transmitted information bits for the CCR can be represented as $R_{\mathrm{PU}} T$

Figure $2 \mathrm{~b}$ depicts the transmission method of NSCRS with NAF relaying, in which the time slot consists of two phases with identical durations and incentive time. In phase 1 , the $\mathrm{PU}_{s}$ sends pilots and information to the $\mathrm{PU}_{d}$. Then the CUs will estimate the energy efficiency by the received pilot and cooperate with $\mathrm{PU}_{s}$ in phase 2 if the energy efficiency of cooperation is better than that of CCR. Because of the higher transmission rate with

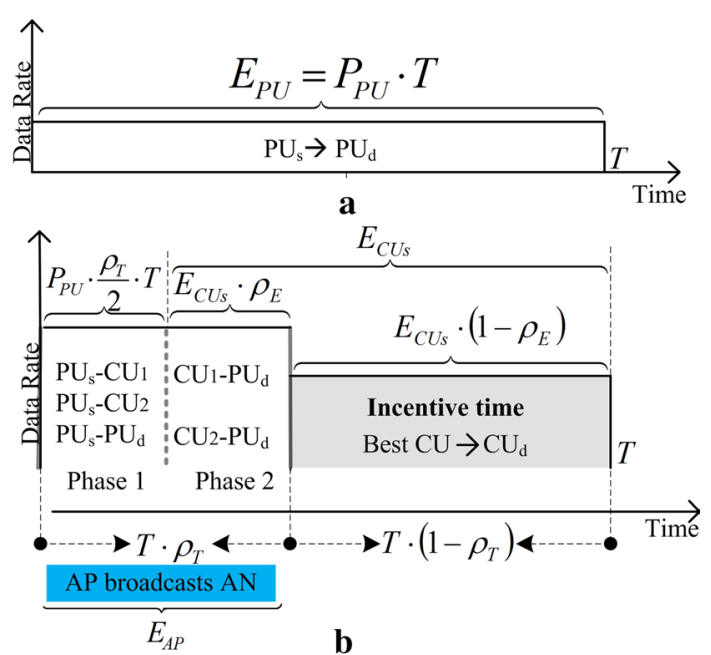

Fig. 2 Transmission methods for CCR and NSCRS 
cooperation, the time consumption of transmission for $\mathrm{PU}_{d}$ can be reduced to $\rho_{T} T$, where $\rho_{T}$ is a time allocation ratio parameter. The rest time $\left(1-\rho_{T}\right) T$ is named as incentive time, in which the CUs can send their own information to the AP. The energy consumption of $\mathrm{PU}_{s}$ is denoted as $E_{\mathrm{PU}} \rho_{T} / 2$ in phase 1 and $E_{\mathrm{CUs}}$ is considered as the total energy constraint of CUs. $E_{\mathrm{CUs}} \rho_{E}$ is the energy consumption of CUs used for cooperation for $\mathrm{PU}_{d}$ in phase 2 and the rest energy of $E_{\mathrm{CUs}}\left(1-\rho_{E}\right)$ at $\mathrm{CUs}$ is utilized to transmit their own information to the $\mathrm{AP}$, where $\rho_{\mathrm{E}}$ is the energy allocation ratio parameter.

Moreover, to prevent information leakage, AP broadcasts two kinds of AN $x_{N}^{1}$ and $x_{N}^{2}$ in phase 1 and phase 2, respectively. Depending on the CSI information, $x_{N}^{1}$ can be successfully eliminated by the $x_{N}^{2}$ at the $\mathrm{PU}_{d}$. The energy consumption of AP during phases 1 and 2 is assumed to be $E_{\mathrm{AP}}$. Thus, the transmit power of AP for AN can be described as $P_{\mathrm{AP}}=E_{\mathrm{AP}} /\left(\rho_{T} T\right)$.

As shown in Fig. $1, \alpha_{k}, \beta_{k}, h_{k}$ represent the channel gains of $\mathrm{PU}_{s}-\mathrm{CU}_{k}, \mathrm{CU}_{k}-\mathrm{PU}_{d}$ and $\mathrm{CU}_{k}$-AP, respectively. Besides, the energy consumption at the $k$ th $\mathrm{CU}$ for the cooperative transmission is denoted by $E_{c u}^{k}$.

In phase 1 , the $\mathrm{PU}_{s}$ transmits a signal to $C U$ s and $\mathrm{PU}_{d}$, and AP broadcasts jamming signal $x_{N}^{1}$. Thus, the signal received at the $k$ th $\mathrm{CU}$ can be given as

$$
y_{c u}^{k}=\sqrt{\frac{E_{\mathrm{PU} \alpha_{k}}}{T}} \cdot x_{S}+\sqrt{P_{\mathrm{AP}} h_{k}} \cdot x_{N}^{1}+n_{k}
$$

where $x_{S}$ denotes the desired signal from $\mathrm{PU}_{s}$ and $n_{k}$ is the AWGN. Thus, the signal received at the $\mathrm{PU}_{d}$ in phase $1, y_{d}^{1}$, can be described as,

$$
y_{d}^{1}=\sqrt{\frac{E_{\mathrm{PU}} \alpha^{\mathrm{PU}}}{T}} \cdot x_{S}+\sqrt{P_{\mathrm{AP}} h^{\mathrm{PU}}} \cdot x_{N}^{1}+n_{d} .
$$

In phase 2, the received signals at CUs are, respectively, amplified and retransmitted to the $\mathrm{PU}_{d}$, where the non-orthogonal $\mathrm{AF}$ relaying is considered and all the CUs are allowed to transmit their signals simultaneously. And AP broadcasts jamming signal $x_{N}^{2}$. In order to remove the effect of $\mathrm{AN}$ at $\mathrm{PU}_{d}, x_{N}^{2}$ is designed as

$$
x_{N}^{2}=-\frac{\sum_{k=1}^{K}\left(\sqrt{\frac{2 E_{c u_{k}} \beta_{k} h_{k}}{\rho_{T} E_{\mathrm{PU}} \alpha_{k}+E_{\mathrm{AP}} h_{k}+\rho_{T} T N}}\right)+\sqrt{h^{\mathrm{PU}}}}{\sqrt{h^{\mathrm{PU}}}} x_{N}^{1} .
$$

And the signal transmitted from the $k$ th $\mathrm{CU}$ to the $\mathrm{PU}_{d}$ can be described by

$$
s_{c u_{k}}=\sqrt{\frac{2 E_{c u_{k}}}{\rho_{T} E_{\mathrm{PU}} \alpha_{k}+E_{\mathrm{AP}} h_{k}+\rho_{T} T N}} \cdot\left(\sqrt{\frac{E_{\mathrm{PU}} \alpha_{k}}{T}} \cdot x_{S}+\sqrt{P_{\mathrm{AP}} h_{k}} \cdot x_{N}^{1}+n_{k}\right) .
$$

Delay diversity can be used to combine the received signals from CUs in phase 2. It is assumed that the transmitted signals from CUs may arrive at the $\mathrm{PU}_{d}$ with different delays. Then, the $\mathrm{PU}_{d}$ can coherently combine the entire received signals along the paths by the Rake receiver with maximum ratio combining (MRC). Thus, the signal received at $\mathrm{PU}_{d}$ in phase 2 can be given by 


$$
\begin{aligned}
y_{d}^{2}= & \sum_{k=1}^{K}\left(\sqrt{\beta_{k}} s_{c u_{k}}+n_{k}^{\prime}\right)+\sqrt{P_{\mathrm{AP}} h^{\mathrm{PU}}} x_{N}^{2}+n_{d}^{\prime} \\
= & \sum_{k=1}^{K}\left(\sqrt{\frac{2 E_{c u_{k}} \beta_{k}}{\rho_{T} E_{\mathrm{PU}} \alpha_{k}+E_{\mathrm{AP}} h_{k}+\rho_{T} T N}}\left(\sqrt{\frac{E_{\mathrm{PU} \alpha_{k}}}{T}} x_{S}+\sqrt{P_{\mathrm{AP}} h_{k}} x_{N}^{1}+n_{k}\right)+n_{k}^{\prime}\right) \\
& +\sqrt{P_{\mathrm{AP}} h^{\mathrm{PU}}} x_{N}^{2}+n_{d}^{\prime} .
\end{aligned}
$$

Thus, based on (5) the total signal received at $\mathrm{PU}_{d}$ can be expressed as

$$
\begin{aligned}
y_{d}= & y_{d}^{1}+y_{d}^{2}=\sqrt{\frac{E_{\mathrm{PU} \alpha^{\mathrm{PU}}}^{T}}{T}} x_{S}+\sqrt{P_{\mathrm{AP}} h^{\mathrm{PU}}} x_{N}^{1}+n_{d} \\
& +\sum_{k=1}^{K}\left(\sqrt{\frac{2 E_{c u_{k}} \beta_{k}}{\rho_{T} E_{\mathrm{PU} \alpha_{k}}+E_{\mathrm{AP}} h_{k}+\rho_{T} T N}}\left(\sqrt{\frac{E_{\mathrm{PU} \alpha_{k}}}{T} x_{S}}+\sqrt{P_{\mathrm{AP}} h_{k}} x_{N}^{1}+n_{k}\right)+n_{k}^{\prime}\right) \\
& +\sqrt{P_{\mathrm{AP}} h^{\mathrm{PU}}} x_{N}^{2}+n_{d}^{\prime} .
\end{aligned}
$$

After substitute (6) into (9), we can get (10), since (11) is satisfied.

$$
\begin{aligned}
& y_{d}= \sqrt{\frac{E_{\mathrm{PU} \alpha^{\mathrm{PU}}}}{T}} \cdot x_{S}+n_{d} \\
&+\sum_{k=1}^{K}\left(\sqrt{\frac{2 E_{c u_{k}} \beta_{k}}{\rho_{T} E_{\mathrm{PU} \alpha_{k}}+E_{\mathrm{AP}} h_{k}+\rho_{T} T N}} \cdot\left(\sqrt{\frac{E_{\mathrm{PU} \alpha_{k}}}{T}} \cdot x_{S}+n_{k}\right)+n_{k}^{\prime}\right)+n_{d}^{\prime} \\
& \sum_{k=1}^{K}\left(\sqrt{\frac{2 E_{c u_{k}} \beta_{k}}{\rho_{T} E_{\mathrm{PU}} \alpha_{k}+E_{\mathrm{AP}} h_{k}+\rho_{T} T N}} \cdot \sqrt{P_{\mathrm{AP}} h_{k}} \cdot x_{N}^{1}\right) \\
&+ \sqrt{P_{\mathrm{AP}} h^{\mathrm{PU}}} \cdot x_{N}^{1}+\sqrt{P_{\mathrm{AP}} h^{\mathrm{PU}}} \cdot x_{N}^{2}=0
\end{aligned}
$$

For the received signal at the undesired nodes, the AN cannot be canceled since channel characteristics are unknown. Thus, the SNR of the undesired nodes is heavily degraded and the privacy preserving can be guaranteed.

Thanks to the excellent autocorrelation property of well-designed spreading code or interference cancellation, the interference at the $\mathrm{PU}_{d}$ from CUs can be neglected. Then, after combining the received signals in phases 1 and 2 with MRC [27], the received SNR at the $\mathrm{PU}_{d}$ with cooperation from CUs, $\gamma^{c u}$, and the corresponding transmission rate, $R^{c u}$, can be, respectively, expressed as

$$
\begin{aligned}
& \gamma^{c u}=\frac{E_{\mathrm{PU}} \alpha^{\mathrm{PU}}}{T N}+\frac{\sum_{k=1}^{K} \frac{E_{\mathrm{PU}}}{T} \cdot \frac{2 E_{c u}^{k}}{\rho_{T} T} \cdot \alpha_{k} \cdot \frac{T}{E_{\mathrm{PU} \alpha_{k}}+P_{\mathrm{AP}} T+T N} \cdot \beta_{k}}{\sum_{k=1}^{K} \frac{2 E_{c u}^{k}}{\rho_{T} T} \cdot \frac{T}{E_{\mathrm{PU} \alpha_{k}}+P_{\mathrm{AP}} T+T N} \cdot \beta_{k} N+N}, \\
& R^{c u}=\frac{1}{2} \cdot \log _{2}\left(1+\gamma_{c u}\right) .
\end{aligned}
$$

The right two terms of (12) represent the received SNR at the $\mathrm{PU}_{d}$ in phase 1 and phase 2 , respectively. Also, the received noises amplified by the CUs still remain in the 
denominator of the second term, while the interference from other CUs is neglected. The coefficient of $1 / 2$ in (13) is caused by the half duplex AF relaying scheme with two identical duration phases.

Owing to the cooperation by the CUs, the required transmission time can be reduced to $\rho_{T} T$ and the rest of original time $\left(1-\rho_{T}\right) T$ will be allocated to CUs as the incentive time. Simultaneously, to get the maximal transmission rate in the incentive time, the incentive time $\left(1-\rho_{T}\right) T$ will be allocated to the best $\mathrm{CU}$, i.e., the hlCU which has the highest SNR to the AP among all the CUs. As a result, the $k_{i}$-th CU is selected based on (14).

$$
k_{1}=\arg \max _{i \in(1,2, . ., K)} \frac{E_{\mathrm{CUs}}\left(1-\rho_{E}\right) h_{i}}{\left(1-\rho_{T}\right) T N},
$$

where $h_{i}, i \in[1,2, \ldots, K]$, represents the channel gain from the $i$-th CU to the AP. Thus, the corresponding transmission rate in the incentive time can be given as

$$
R_{\text {inc }}=\log _{2}\left(1+\frac{E_{\mathrm{CUs}}\left(1-\rho_{E}\right) h_{k_{1}}}{\left(1-\rho_{T}\right) T N}\right) .
$$

\section{Problem formulation}

\subsection{Energy efficiency of NSCRS}

As a reward, an incentive time will be allocated to CUs if the cooperative transmission by CUs can offer a higher energy efficiency for the system. Otherwise, the $P U_{s}$ will occupy the entire duration and no incentive time will be allocated to the CUs, i.e., CCR. So the energy efficiency, which is defined as the total number of transmitted bits divided by the total consumed energy, in NSCRS can be given as

$$
\eta=\max \left(\eta^{\mathrm{PU}}, \eta^{c u}\right)
$$

where the function $\max (\cdot, \cdot)$ will return the maximum value of the arguments. $\eta^{\mathrm{PU}}$ and $\eta^{c u}$, respectively, represent the energy efficiency of CCR and NSCRS. $\eta^{\mathrm{PU}}$ and $\eta^{c u}$ can be given as

$$
\begin{aligned}
\eta^{\mathrm{PU}} & =R_{\mathrm{PU}} /\left(E_{\mathrm{PU}}+E_{\mathrm{AP}}\right), \\
\eta^{c u} & =\frac{\frac{1}{2} \log _{2}\left(1+\gamma^{c u}\right) \cdot \rho_{T} T+R_{i n c} \cdot\left(1-\rho_{T}\right) T}{E_{\mathrm{PU}} \cdot \frac{\rho_{T}}{2}+E_{\mathrm{CUs}}+E_{\mathrm{AP}}} .
\end{aligned}
$$

The denominator of (18) represents the total consumed energy of NSCRS and the numerator of (18) is the total throughput of both primary and cognitive networks.

\subsection{Problem formulation for NSCRS with privacy preserving}

In this paper, we aim to maximize the energy efficiency of NSCRS through optimally allocating the time and energy resource of $\mathrm{PU}_{s}$ and CUs. According to (18), the energy efficiency optimization problem of NSCRS can be formulated as 


$$
\begin{aligned}
& \max _{\rho_{T}, \rho_{E}, E_{c u}^{k}: k=1, \cdots, K} \frac{\frac{1}{2} \log _{2}\left(1+\gamma^{c u}\right) \cdot \rho_{T} T+R_{\text {inc }} \cdot\left(1-\rho_{T}\right) T}{E_{\mathrm{PU}} \cdot \frac{\rho_{T}}{2}+E_{\mathrm{CUs}}+E_{\mathrm{AP}}}, \\
& \text { s.t. } \frac{\frac{1}{2} \log _{2}\left(1+\gamma^{c u}\right) \cdot \rho_{T} T+R_{i n c} \cdot\left(1-\rho_{T}\right) T}{E_{\mathrm{PU}} \cdot \frac{\rho_{T}}{2}+E_{\mathrm{CUs}}+E_{\mathrm{AP}}} \\
& \quad>\frac{\log _{2}\left(1+\gamma_{\mathrm{PU}}\right) \cdot T}{E_{\mathrm{PU}}}, \\
& \frac{1}{2} \log _{2}\left(1+\gamma^{c u}\right) \cdot \rho_{T} T \geq \log _{2}\left(1+\frac{E_{\mathrm{PU}} \alpha^{\mathrm{PU}}}{T N}\right) \cdot T, \\
& \sum_{k=1}^{K} E_{c u}^{k} \leq \rho_{E} E_{\mathrm{CUs}}, E_{c u}^{k} \geq 0 .
\end{aligned}
$$

The objective function in (19a) intends to maximize the energy efficiency of NSCRS by optimally allocating the energy of CUs and time of $\mathrm{PU}_{s}$ for the data transmission to the $\mathrm{PU}_{d}$. The constraint in (19b) means that the energy efficiency of NSCRS should be better than that of CCR. Constraint in (19c) implies that the total transmission bits of NSCRS should be larger than that of CCR. Constraint in (19d) denotes the summed as well as the individual power constraint of CUs. With the fixed $\rho_{E}$ and $\rho_{T}$, we can get the maximum of EE for NSCRS as (20a)-(20d), and the related Proof is given in "Appendix".

$$
\begin{aligned}
& \frac{\frac{1}{2} \log _{2}\left(1+\gamma^{c u^{\prime}}\right) \cdot \rho_{T} T+\log _{2}\left(1+\frac{E_{\mathrm{CUs}}\left(1-\rho_{E}\right) h_{k_{1}}}{\left(1-\rho_{T}\right) T N}\right) \cdot\left(1-\rho_{T}\right) T}{E_{\mathrm{PU}} \cdot \frac{\rho_{T}}{2}+E_{\mathrm{CUs}}+E_{\mathrm{AP}}}, \\
& \text { s.t. } \frac{\frac{1}{2} \log _{2}\left(1+\gamma^{c u^{\prime}}\right) \cdot \rho_{T} T+\log _{2}\left(1+\frac{E_{\mathrm{CUs}}\left(1-\rho_{E}\right) h_{k_{1}}}{\left(1-\rho_{T}\right) T N}\right) \cdot\left(1-\rho_{T}\right) T}{E_{\mathrm{PU}} \cdot \frac{\rho_{T}}{2}+E_{\mathrm{CUs}}+E_{\mathrm{AP}}} \\
& \quad>\frac{\log _{2}\left(1+\gamma_{\mathrm{PU}}\right) \cdot T}{E_{\mathrm{PU}}}, \\
& \frac{1}{2} \log _{2}\left(1+\gamma^{c u^{\prime}}\right) \cdot \rho_{T} T \geq \log _{2}\left(1+\frac{E_{\mathrm{PU}} \alpha_{m}^{\mathrm{PU}}}{T N}\right) \cdot T, \\
& E_{\mathrm{CUs}} \geq 0 .
\end{aligned}
$$

where

$$
\gamma^{c u^{\prime}}=\frac{E_{\mathrm{PU}} \alpha^{\mathrm{PU}}}{T N}+\frac{\frac{E_{\mathrm{PU}}}{T} \cdot \frac{2 \rho_{E} E_{\mathrm{CUs}}}{\rho_{T} T} \cdot \alpha_{k^{\prime}} \cdot \frac{T}{E_{\mathrm{PU} \alpha_{k^{\prime}}}+P_{\mathrm{AP}} T+T N} \cdot \beta_{k^{\prime}}}{\frac{2 \rho_{E} E_{\mathrm{CUs}}}{\rho_{T} T} \cdot \frac{T}{E_{\mathrm{PU} \alpha_{k^{\prime}}}+P_{\mathrm{AP}} T+T N} \cdot \beta_{k^{\prime}} N+N},
$$

and 


$$
k^{\prime}=\arg \max _{k=1,2, \ldots, K} \frac{\frac{E_{\mathrm{PU}}}{T} \cdot \frac{2 \rho_{E} E_{\mathrm{CU}}}{\rho_{T} T} \cdot \alpha_{k} \cdot \frac{T}{E_{\mathrm{PU} \alpha_{k}}+P_{\mathrm{AP}} T+T N} \cdot \beta_{k}}{\frac{2 \rho_{E} E_{\mathrm{CUs}}}{\rho_{T} T} \cdot \frac{T}{E_{\mathrm{PU}} \alpha_{k}+P_{\mathrm{AP}} T+T N} \cdot \beta_{k} N+N} .
$$

According to (16), we can decompose the optimization problem into two cases.

Case 1. Consider $\eta=\eta^{\mathrm{PU}}$ as $\mathrm{PU}_{s}$ transmits the signals to the $\mathrm{PU}_{d}$ without the cooperation from CUs as in CCR.

Case 2. Consider $\eta=\eta^{c u}$ as $\mathrm{PU}_{s}$ transmits the signals to the $\mathrm{PU}_{d}$ with the cooperation of CUs.

\section{Proposed energy efficiency algorithm with full or partial CSI}

If the CSI of $\alpha_{k}$ and $\beta_{k}$ can be estimated by the CUs, then the full CSI of $\alpha^{\mathrm{PU}}, \alpha_{k}$, and $\beta_{k}$ will be available for the AP, which is called "Scenario 1" for NSCRS. Nevertheless, instantaneous $\beta_{k}$ may not be available at the AP. Thus, we further consider a "Scenario 2" for the NSCRS with only the partial CSI, that is, $\alpha^{\mathrm{PU}}, \alpha_{k}$ and an averaged CSI of by long-term observation rather than an instantaneous value. In this section, the energy and time allocation algorithms for both scenarios are investigated. In addition, an equal energy allocation algorithm (EPA), in which the total available energy of the CUs is equally distributed to the CUs, that is, $E_{c u}^{k}=E_{\mathrm{CUs}} \cdot \rho_{E} / K$, is also considered.

\subsection{Optimal energy and time allocation algorithm with full CSI}

With full CSI, we can obtain the optimal solution of $\rho_{T}=0$ and $\rho_{E}=0$ for case 1 . And for case 2, the optimal algorithm is proposed as follows, by which the optimal $\rho_{T}$ and $\rho_{E}$ can be obtained.

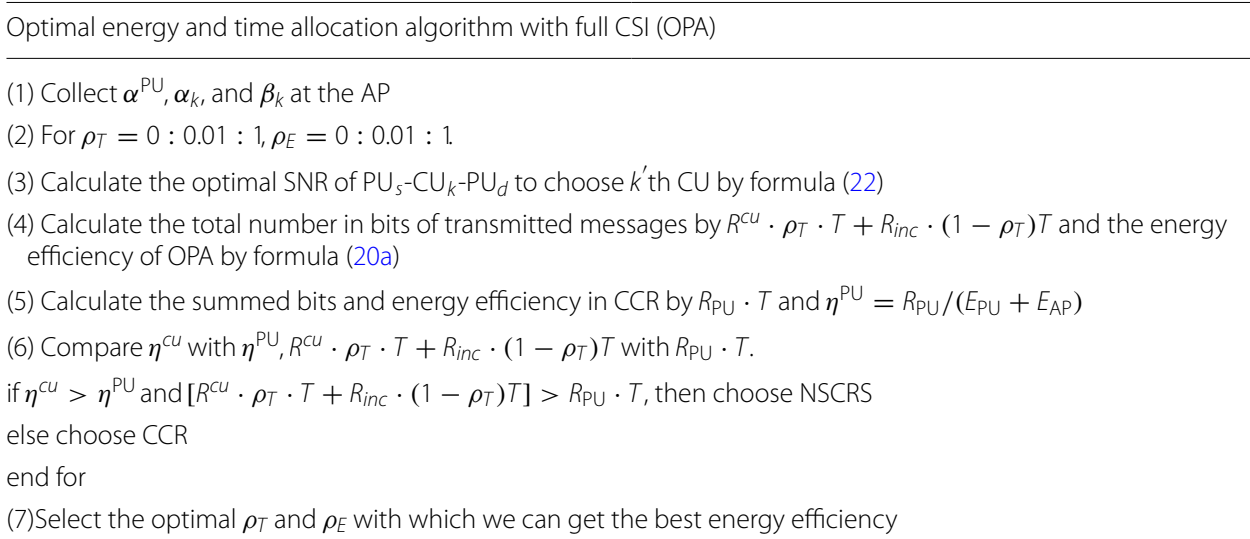

Firstly, the CSI of $\alpha^{\mathrm{PU}}, \alpha_{k}$ and $\beta_{k}$ is collected at the AP. Then, we calculate the optimal SNR of $\mathrm{PU}_{s}-\mathrm{CUk}-\mathrm{PU}_{d}$ to choose $k^{\prime}$ th $\mathrm{CU}$ by formula (22) with given pair of $\rho_{T}$ and $\rho_{E}$ in the range of $[0,1]$. Then, the total number in bits of transmitted messages and energy efficiency of OPA and CCR will be calculated, respectively. If the transmitted information bits as well as the energy efficiency of OPA are both larger than these of CCR, the NSCRS will be chosen. Otherwise, the CCR will be chosen. Finally, the optimal $\rho_{T}$ and $\rho_{E}$ can be obtained for the best energy efficiency. 


\subsection{Suboptimal energy and time allocation algorithm with partial CSI feedback}

Although OPA is able to achieve the optimal solution in scenario 1, the full instantaneous CSI feedback is needed. Sometimes it is hard to get the feedback of $\beta_{k}$ immediately. For such case, it is assumed that the channel quality of CUs-PU ${ }_{d}$ is much better than that of $\mathrm{PU}_{s}$-CUs, i.e., $P_{C} \cdot \beta_{k}>>P_{\mathrm{PU}} \cdot \alpha_{k}$ and $P_{C} \cdot \beta_{k}>>N$, then $2 \rho_{E} E_{\mathrm{CUs}} \beta_{k} /\left(\rho_{T} T\right)>>E_{\mathrm{PU}} \alpha_{k} / T$ and $2 \rho_{E} E_{\mathrm{CUs}} \beta_{k} /\left(\rho_{T} T\right)>>N$ can be satisfied. Thus, $x_{N}^{2}$ can be described as

$$
x_{N}^{2}=-\frac{\sum_{k=1}^{K}\left(\sqrt{\frac{2 E_{c u_{k}} \beta_{k}}{E_{\mathrm{AP}}}}\right)+\sqrt{h^{\mathrm{PU}}}}{\sqrt{h^{\mathrm{PU}}}} x_{N}^{1} .
$$

However, due to the lack of $\beta_{k}, x_{N}^{2}$ is hard to be perfectly eliminated at the $\mathrm{PU}_{d}$. Rather than considering an instantaneous value of $\beta_{k}$, an averaged value of $\bar{\beta}_{k}$, which could be obtained by a long-term observation, is assumed to be adopted. Then, the interference power introduced by the AN can be given as $2 E_{c u_{k}}\left|\beta_{k}-\overline{\beta_{k}}\right| /\left(\rho_{T} T\right)$. Therefore, the received $\mathrm{SNR}$ at $\mathrm{PU}_{d}$ with cooperation from $\mathrm{CUs}, \gamma_{c u}$, can be transformed to

$$
\gamma_{c u}=\frac{E_{\mathrm{PU}} \alpha^{\mathrm{PU}}}{T N}+\frac{\frac{E_{\mathrm{PU}}}{T} \alpha_{k}}{N+\frac{2 E_{c u_{k}}\left|\beta_{k}-\bar{\beta}\right|}{\rho_{T} T}} .
$$

Since the i.i.d slow block fading channel is considered in this paper, $\beta_{k} \approx \overline{\beta_{k}}$ can be satisfied during each fading block. Compared with the case with instantaneous value of $\beta_{k}, \overline{\beta_{k}}$ is more easy to obtained via a long-term observation, and thus, the CSI feedback overhead of $\beta_{k}$ can be obviously reduced during each fading block. So the $\gamma_{c u}$ and the index of the selected CUs for incentive time can be, respectively, transformed to

$$
\begin{aligned}
& \gamma_{c u}=\frac{E_{\mathrm{PU}} \alpha^{\mathrm{PU}}}{T N}+\frac{E_{\mathrm{PU}} \alpha_{k}}{T N}, \\
& k^{\prime \prime}=\arg \max _{k=1,2, \ldots, K} \frac{E_{\mathrm{PU} \alpha_{k}}}{T N} .
\end{aligned}
$$

Here, we define a ratio parameter, $\theta=\alpha_{k} / \alpha^{\mathrm{PU}}$, to decide whether the $\alpha_{k}$ is much better than $\alpha^{\mathrm{PU}}$ or not and the suboptimal algorithm for partial CSI feedback can be given as below.

Partial CSI feedback based suboptimal energy and time allocation algorithm (PPA)

(1) Collect $\alpha^{\mathrm{PU}}, \alpha_{k}$ and $\overline{\beta_{k}}$ at the AP

(2) For $\rho_{T}=0: 0.01: 1, \rho_{E}=0: 0.01: 1$

(3) Decide the optimal method by comparing $\theta$ with $\theta_{t h}$, where $\theta_{\text {th }}$ is a predetermined threshold for deciding whether the cooperation from CUs to the $\mathrm{PU}_{d}$ is effective or not

i)if $\theta<\theta_{\text {th }}$, choose CCR

ii)if $\theta>\theta_{t h}$, calculate the parameters as follows

(4) Calculate the total number in bits of transmitted messages and the energy efficiency of both PPA and CCR by the $k^{\prime \prime}$ th $\mathrm{CU}$

(5) Compare $\eta^{\text {cu }}$ with $\eta^{\mathrm{PU}}, R^{c u} \cdot \rho_{T} \cdot T+R_{\text {inc }} \cdot\left(1-\rho_{T}\right) T$ with $R_{\mathrm{PU}} \cdot T$

if $\eta^{c u}>\eta^{\mathrm{PU}}$ and $\left[R^{c u} \cdot \rho_{T} \cdot T+R_{\text {inc }} \cdot\left(1-\rho_{T}\right) T\right]>R_{\mathrm{PU}} \cdot T$, then choose NSCRS 
Partial CSI feedback based suboptimal energy and time allocation algorithm (PPA)

else choose CCR

end for

(6)Select the optimal $\rho_{T}$ and $\rho_{E}$ with which we can get the best energy efficiency

Firstly, the CSI of $\alpha^{\mathrm{PU}}, \alpha_{k}$, and $\overline{\beta_{k}}$ are collected at the AP, where $\overline{\beta_{k}}$ is not needed to be feedback during a fading block. Then, $\theta$ and $\theta_{t h}$ are compared with each other to decide the optimal method. if $\theta<\theta_{t h}$ is satisfied, the CCR will be chosen. Otherwise, we further calculate the total number of bits of transmitted messages as well as the energy efficiency for both PPA and CCR. If the summed bits and energy efficiency of OPA are both larger than these of CCR, the NSCR will be selected. Otherwise, the CCR will be chosen. Finally, the optimal $\rho_{T}$ and $\rho_{E}$ can be obtained.

\section{Methods}

Figure 3 shows the simulation model for the networks of PUs, CUs, and AP. $\mathrm{PU}_{s}, \mathrm{PU}_{d}$, $\mathrm{AP}$ and CUs are placed within a 2-dimensional area $(500 \mathrm{~m} * 500 \mathrm{~m}) . \mathrm{PU}_{s}$ and $\mathrm{PU}_{d}$ are, respectively, fixed at $(0,250)$ and $(500,250) .10 \mathrm{CUs}$ are randomly placed within this region. In addition, we place a AP at $(0,0)$. A simple pass loss model of $1 / d^{3}$, where $d$ is the distance between two points, is considered. Block Rayleigh fading channels are assumed among $\mathrm{PU}_{s}, \mathrm{CUs}, \mathrm{PU}_{d}$, and AP. The AWGN power in this region is assumed to be $-50 \mathrm{dBm}$ and the $P_{\mathrm{AP}}$ is set to be $15 \mathrm{dBm}$. We consider energy with a unit $\mathrm{dBJ}$, where $\mathrm{dBJ}=10 \log _{10} \mathrm{~J}$. The data to be transmitted from $\mathrm{PU}_{s}$ to $\mathrm{PU}_{d}$ are assumed large enough to guarantee the full time transmission between $\mathrm{PU}_{s}-\mathrm{PU}_{d}$ in case of CCR. Therefore, CUs have no chance to access the AP in the case of CCR. In addition $\theta_{t h}$ is with a range of $\left[10^{-10}<\theta_{t h}<10^{10}\right]$ for a given pair of $\left(\rho_{T}, \rho_{E}\right)$.

\section{Simulation results and discussion}

Figure 4 shows the energy efficiency for NSCRS with OPA, PPA, and EPA compared to CCR under the constraint of $P_{\mathrm{PU}}=30 \mathrm{dBm}, E_{\mathrm{CUs}}=0 \mathrm{dBJ}$.The CCR has the lowest EE compared to the OPA, PPA, and EPA. OPA always outperforms the others owning to the

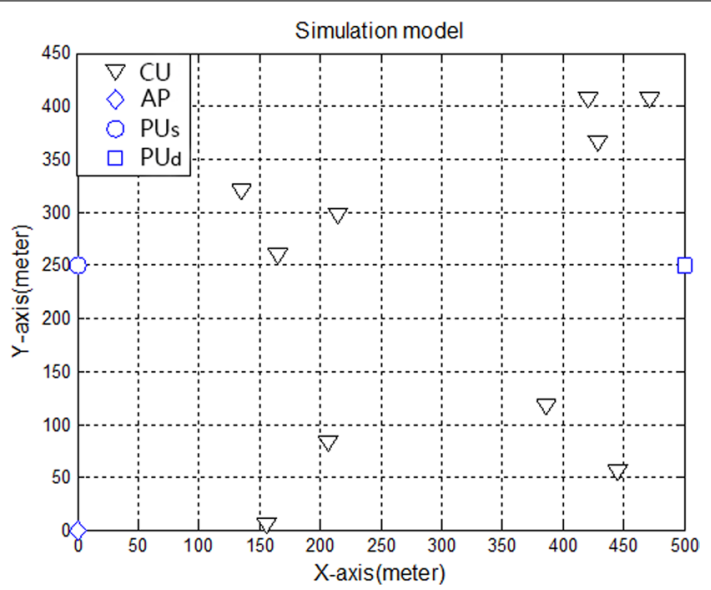

Fig. 3 The simulation model of NSCRS 


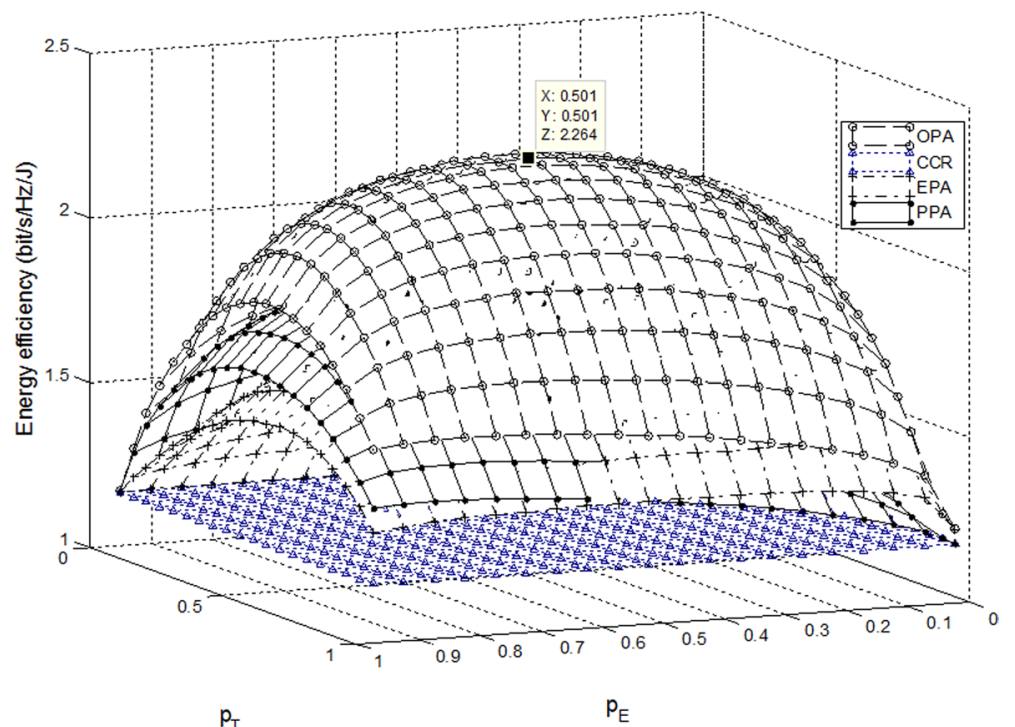

Fig. 4 Energy efficiency for NSCRS with OPA, PPA, and EPA compared to CCR
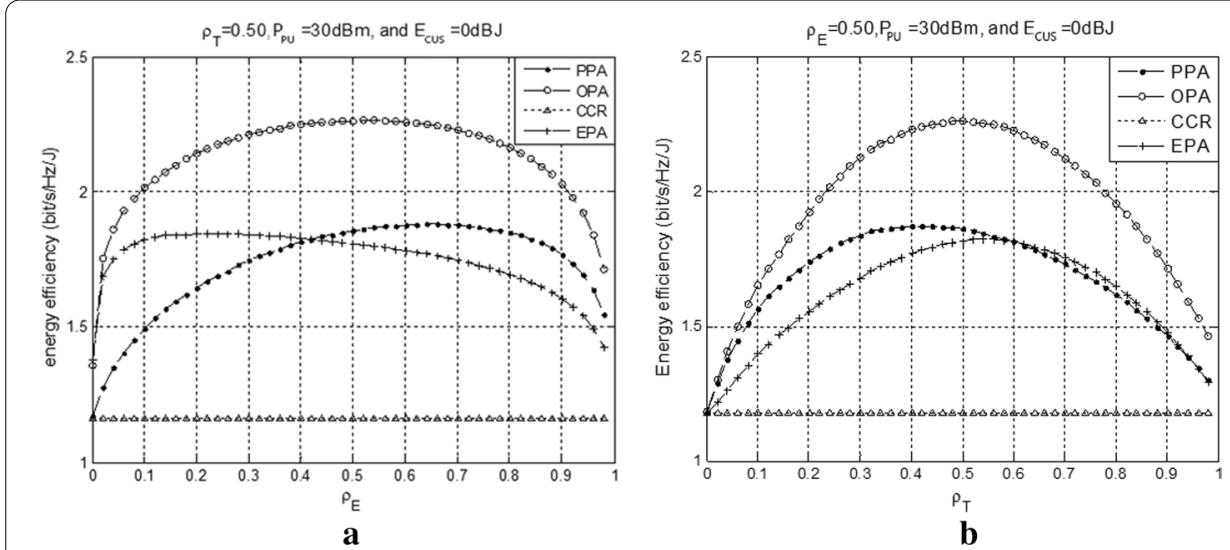

Fig. 5 Energy efficiency for different algorithms as a function of $\rho_{E}$ and $\rho_{T}$, respectively

full CSI feedback and an optimal EE of NSCRS can be achieved at $x=0.501, y=0.501$, and $\mathrm{z}=2.264$. Compared with the OPA, a small performance gap can be observed between OPA and PPA, since only partial CSI is fed back for the PPA rather than the full CSI feedback of OPA.

Figure $5 \mathrm{a}, \mathrm{b}$ describe the energy efficiency for different algorithms as a function of $\rho_{E}$ and $\rho_{T}$, respectively. As $\rho_{E}$ changes in Fig. 5a, the energy efficiencies of OPA, PPA and EPA increase at first, since the cooperation from CUs is effective. However, when $\rho_{E}$ becomes very large, the transmission rate for CU's data transmission becomes worse, which results in a decrease in terms of overall energy efficiency. In addition, when $\rho_{E}$ is small, EPA performs better than PPA, because condition that $2 \rho_{E} E_{\mathrm{CUs}} \beta_{k} /\left(\rho_{T} T\right)>>E_{\mathrm{PU}} \alpha_{k} / T$ is not satisfied. However, when $\rho_{E}$ becomes larger, the condition of $2 \rho_{E} E_{\mathrm{CUs}} \beta_{k} /\left(\rho_{T} T\right)>>E_{\mathrm{PU}} \alpha_{k} / T$ is satisfied and the performance of PPA becomes better than that of EPA. And as $\rho_{T}$ changes in Fig. $5 \mathrm{~b}$, the energy efficiencies 
of OPA, PPA and EPA increase at first owing to the transmission time allocated to PUs. However, when $\rho_{T}$ becomes large enough, the energy efficiencies decrease, because that there is too little incentive time allocated to CUs. Besides, when $\rho_{T}$ is small, PPA performs better than EPA, because condition that $2 \rho_{E} E_{\mathrm{CUs}} \beta_{k} /\left(\rho_{T} T\right)>>E_{\mathrm{PU}} \alpha_{k} / T$ is satisfied. However, with the increase in $\rho_{T}$, that condition is not satisfied, which results that EPA performs better than PPA.

Figure 6 shows the energy efficiency for different algorithms as a function of $P_{\mathrm{PU}}$ under constraints of $\rho_{E}=\rho_{T}=0.5$ and $E_{\mathrm{CUs}}=0 \mathrm{dBJ}$. The OPA always performs better than PPA and EPA owning to the full CSI feedback. When $P_{\mathrm{PU}}$ is small, OPA, EPA and PPA can perform the transmission with the aid of CUs, which results in better performances compared to that of CCR. When $E_{\mathrm{PU}}$ is smaller than $E_{\mathrm{CUs}}$, the condition of $2 \rho_{E} E_{\mathrm{CUs}} \beta_{k} /\left(\rho_{T} T\right)>>E_{\mathrm{PU}} \alpha_{k} / T$ is satisfied, so the performance of PPA is better than that of EPA. However, the condition of $2 \rho_{E} E_{\mathrm{CUs}} \beta_{k} /\left(\rho_{T} T\right)>>E_{\mathrm{PU}} \alpha_{k} / T$ cannot be satisfied when $E_{\mathrm{PU}}$ is larger than $E_{\mathrm{CUs}}$, and a better performance of EPA can be observed compared to the PPA. Moreover, when $E_{\mathrm{PU}}$ becomes large enough, CUs are not needed for cooperation, thus the EE of OPA, PPA, and EPA becomes the same, i.e., $P_{\mathrm{PU}} \geq 50 \mathrm{dBm}$.

Figure 7 shows the energy efficiency for different algorithms as a function of under constraints of $\rho_{E}=\rho_{T}=0.5$ and $E_{\mathrm{PU}}=0 \mathrm{dBJ}$. When $E_{\mathrm{CUs}}$ is small, none of CUs can be utilized for the PU transmission in the cases of OPA, EPA and PPA. Thus, a similar EE can be observed among them. As the $E_{\mathrm{CUs}}$ increases, OPA, EPA and PPA perform better than CCR owing to the assistance of CUs. When $E_{\mathrm{CUs}}$ becomes much larger than that of PUs, the condition of $2 \rho_{E} E_{\mathrm{CUs}} \beta_{k} /\left(\rho_{T} T\right)>>E_{\mathrm{PU}} \alpha_{k} / T$ is satisfied, which results in a better performance of PPA than that of EPA and the performance of PPA becomes similar to that of OPA. However, when $E_{\mathrm{CUs}}$ becomes large enough, e.g., $E_{\mathrm{CUs}} \geq 15 \mathrm{dBJ}$, the performances of OPA, PPA and EPA will decrease to the level of CCR, since the energy consumption of CUs is too big to decrease the EE.

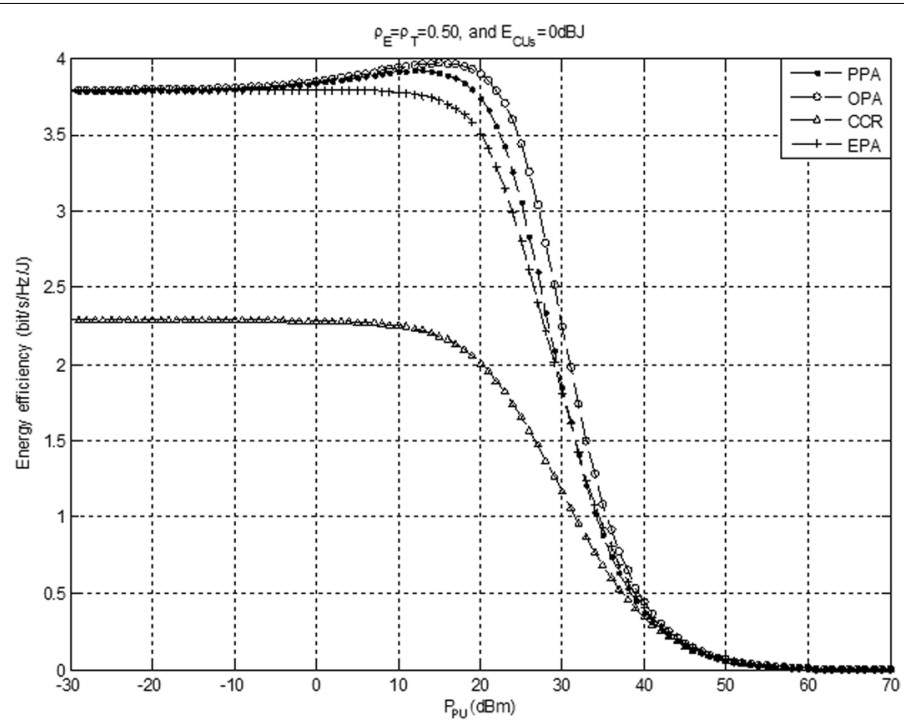

Fig. 6 Energy efficiency for different algorithms as a function of $P_{\mathrm{PU}}$ 


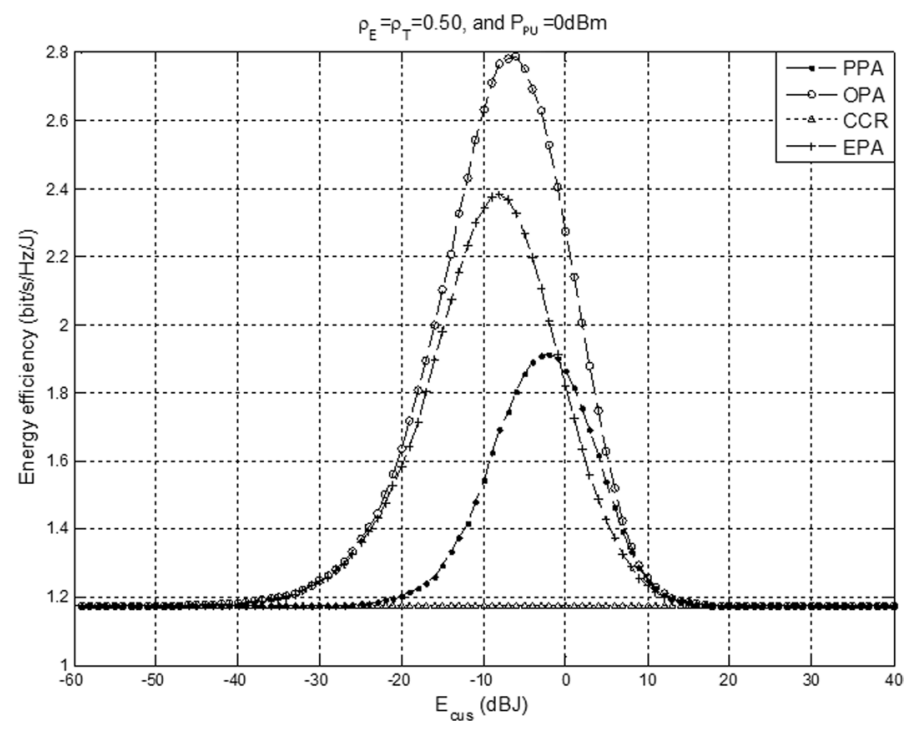

Fig. 7 Energy efficiency for different algorithms as a function of $E_{C} U_{s}$

Figure 8 compares the normalized performance gain of OPA and PPA with an increase trend of $E_{\mathrm{CUs}}$. For a better realization, the performance of EE for both OPA and PPA are normalized by that of CCR. When $E_{\mathrm{CUs}}$ is very small, there is a big gap of performance gain between OPA and PPA as shown in Fig. 8a, b, since the condition of $2 \rho_{E} E_{\mathrm{CUs}} \beta_{k} /\left(\rho_{T} T\right)>>E_{\mathrm{PU}} \alpha_{k} / T$ is not satisfied. However, as the $E_{\mathrm{CUs}}$ increases, e.g., $E_{\mathrm{CUs}}=20 \mathrm{dBJ}$, the approximation of $2 \rho_{E} E_{\mathrm{CUs}} \beta_{k} /\left(\rho_{T} T\right)>>E_{\mathrm{PU}} \alpha_{k} / T$ can be almost achieved. Thus, similar performance gains of OPA and PPA can be observed as shown in Fig. 8e, f. It implies that if the link quality from CUs to the $\mathrm{PU}_{d}$ is much better than those from $\mathrm{PU}_{s}$ to $\mathrm{CUs}$, then the proposed PPA could be an alternative choice with lower instantaneous CSI feedback.

\section{Conclusions}

In this paper, the optimal energy and time allocation algorithm in NSCRS with consideration of privacy preserving was first investigated for energy efficiency maximization in the case of full CSI. To further reduce the overhead from CSI exchanging, a suboptimal energy and time allocation algorithm, where the instantaneous CSI from CUs to PU is not required to be fed back, is alternatively introduced. Simulation results demonstrated that the energy efficiency of primary and cognitive users in the NSCRS can be greatly improved by the proposed OPA and PPA algorithms with the consideration of privacy preserving. Moreover, compared with the OPA, the PPA could achieve a similar performance as that of OPA with a smaller CSI feedback overhead. 


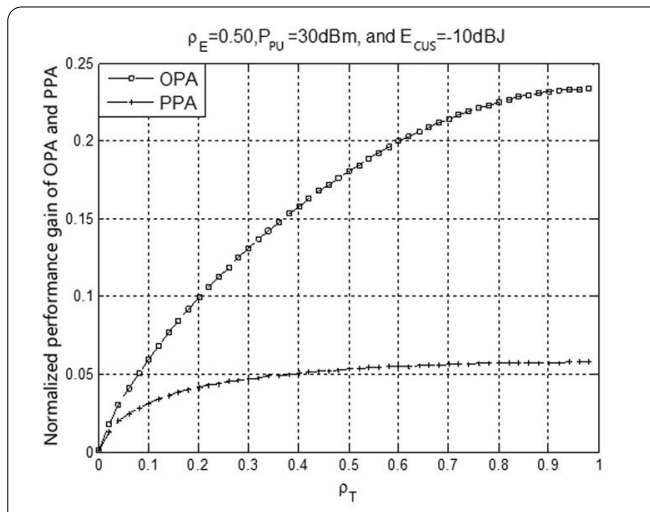

a
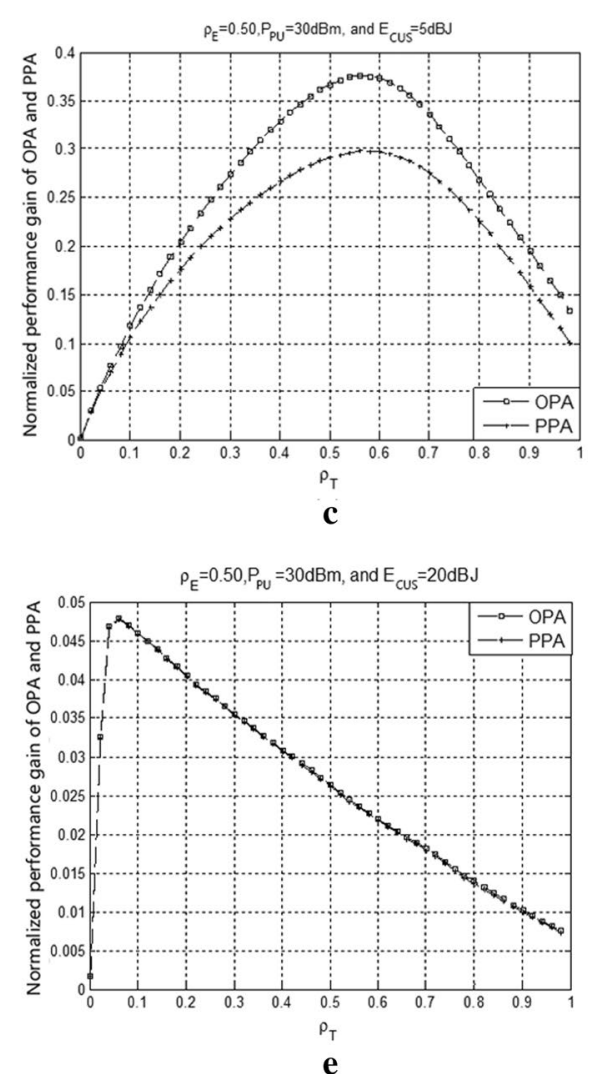

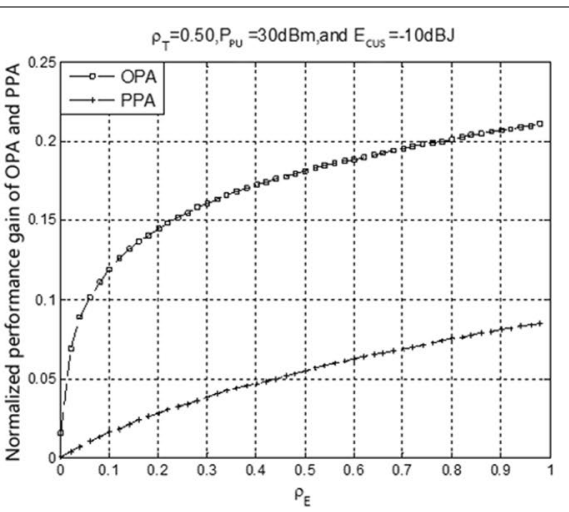

b
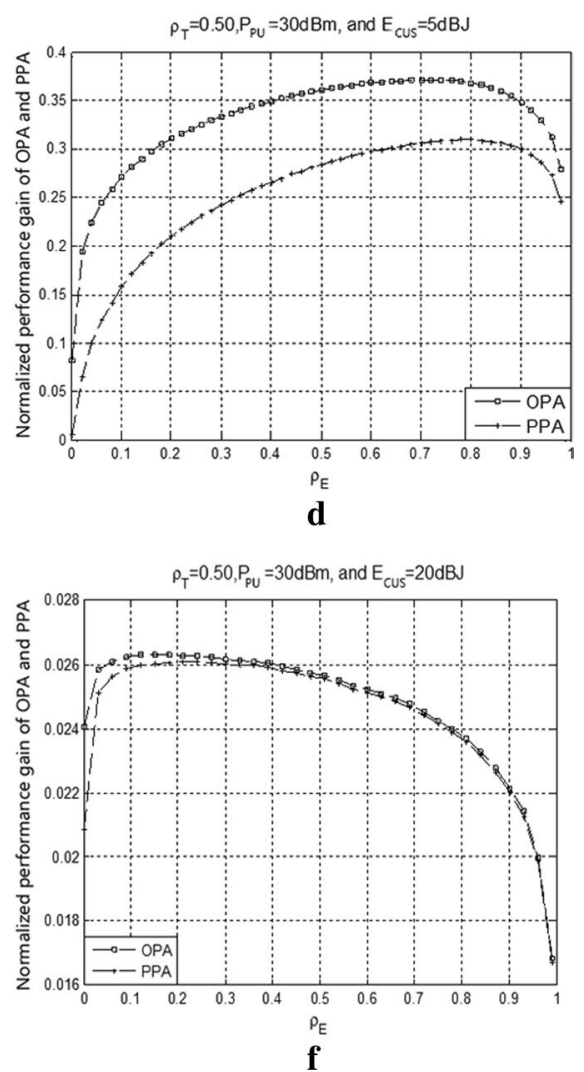

Fig. 8 Performance gain of EE normalized by that of CCR for different algorithms as a function of $\rho_{E}$ and $\rho_{T}$, respectively

\section{Appendix}

First, when we fix $\rho_{T}$ and $\rho_{E}$ the problem can be rewritten in the following form:

$$
\max _{E_{c u}^{k}, k=1, \cdots, K} \frac{\frac{1}{2} \log _{2}(1+A+L) \cdot \rho_{T} T+B}{C+D},
$$

where $\mathrm{A}, \mathrm{B}, \mathrm{C}$ and $\mathrm{D}$ are all nonnegative constants. 


$$
L=\frac{\sum_{k=1}^{K} \frac{E_{\mathrm{PU}}}{T} \cdot \frac{2 E_{c u}^{k}}{\rho_{T} T} \cdot \alpha_{m, k} \cdot \frac{T}{E_{\mathrm{PU} \alpha_{m, k}}+P_{\mathrm{AP}} T+T N} \cdot \beta_{m, k}}{\sum_{k=1}^{K} \frac{2 E_{c u}^{k}}{\rho_{T} T} \cdot \frac{T}{E_{\mathrm{PU}} \alpha_{m, k}+P_{\mathrm{AP}} T+T N} \cdot \beta_{m, k} N+N} .
$$

Obviously, due to the monotonicity of $\log _{2}(x)$ with respect to $x$, the problem in (27) can be converted to maximizing $L$, where

$$
\max L=\max _{E_{c u}^{k}} \frac{\sum_{k=1}^{K} \frac{E_{\mathrm{PU}}}{T} \cdot \frac{2 E_{c u}^{k}}{\rho_{T} T} \cdot \alpha_{m, k} \cdot \frac{T}{E_{\mathrm{PU} \alpha_{m, k}}+P_{\mathrm{AP}} T+T N} \cdot \beta_{m, k}}{\sum_{k=1}^{K} \frac{2 E_{c u}^{k}}{\rho_{T} T} \cdot \frac{T}{E_{\mathrm{PU}} \alpha_{m, k}+P_{\mathrm{AP}} T+T N} \cdot \beta_{m, k} N+N} .
$$

We can rewrite (29) as

$$
\max _{E_{c u}^{k}} f\left(E_{c u}^{1}, E_{c u}^{2}, \ldots, E_{c u}^{K}\right)=\max _{E_{c u}^{k}} \frac{a_{0}+\sum_{k=1}^{K} a_{k} E_{c u}^{k}}{b_{0}+\sum_{k=1}^{K} b_{k} E_{c u}^{k}}
$$

where $a_{0}, a_{k}$ and $b_{k}$ are nonnegative coefficients, but $b_{0}$ is a positive coefficient. Then, we only need to prove the equation as follows

$$
\max _{E_{c u}^{k}} \frac{a_{0}+\sum_{k=1}^{K} a_{k} E_{c u}^{K}}{b_{0}+\sum_{k=1}^{K} b_{k} E_{c u}^{K}}=\max _{k=1,2 \ldots, K} \frac{a_{0}+a_{k} E_{\mathrm{CUs}}}{b_{0}+b_{k} E_{\mathrm{CUs}}} .
$$

We prove (31) by means of the mathematical induction.

Firstly, in the case of $K=2$, we will prove the following equation

$$
\max _{E_{c u}^{k}} \frac{a_{0}+a_{1} E_{c u}^{1}+a_{2} E_{c u}^{2}}{b_{0}+b_{1} E_{c u}^{1}+b_{2} E_{c u}^{2}}=\max \left(\frac{a_{0}+a_{1} E_{\mathrm{CUs}}}{b_{0}+b_{1} E_{\mathrm{CUs}}}, \frac{a_{0}+a_{2} E_{\mathrm{CUs}}}{b_{0}+b_{2} E_{\mathrm{CUs}}}\right) .
$$

It indicates that the maximum is obtained at either $\left(E_{c u}^{1}, E_{c u}^{2}\right)=\left(0, E_{\mathrm{CUs}}\right)$ or $\left(E_{c u}^{1}, E_{c u}^{2}\right)=\left(E_{\mathrm{CUs}}, 0\right)$. By plugging $E_{c u}^{2}=E_{\mathrm{CUs}}-E_{c u}^{1}$ into $f\left(E_{c u}^{1}, E_{c u}^{2}, \ldots, E_{c u}^{K}\right)$ for $K=2$, we get the equivalent form

$$
g\left(E_{c u}^{1}\right)=f\left(E_{c u}^{1}, E_{\mathrm{CUs}}-E_{c u}^{2}\right)=\frac{E_{c u}^{1}\left(a_{1}-a_{2}\right)+E_{\mathrm{CUs}} a_{2}+a_{0}}{E_{c u}^{1}\left(b_{1}-b_{2}\right)+E_{\mathrm{CUs}} b_{2}+b_{0}} .
$$

Therefore, (33) has the maximum at one of the boundary points $E_{c u}^{1}=0$ or $E_{c u}^{1}=E_{\mathrm{CUs}}$ as long as we prove the monotonicity of $g\left(E_{c u}^{1}\right)$. We differentiate $g\left(E_{c u}^{1}\right)$ with respect to $E_{c u}^{1}$

$$
\frac{d g\left(E_{c u}^{1}\right)}{d E_{c u}^{1}}=\frac{\left(a_{1}-a_{2}\right)\left(E_{\mathrm{CUs}} b_{2}+b_{0}\right)-\left(b_{1}-b_{2}\right)\left(E_{\mathrm{CUs}} a_{2}+a_{0}\right)}{\left(E_{c u}^{1}\left(b_{1}-b_{2}\right)+E_{\mathrm{CUs}} b_{2}+b_{0}\right)^{2}} .
$$

The denominator of (34) is constant positive. Hence, the monotonicity of $g\left(E_{c u}^{1}\right)$ depends on the sign of the numerator, which proves (32).

We assume that (31) is true of $K=L$, and we prove that (32) is also true of $K=L+1$. For $K=L+1$, we have

$$
f\left(E_{c u}^{1}, E_{c u}^{2}, \ldots, E_{c u}^{L}, E_{c u}^{L+1}\right)=\frac{a_{0}+\sum_{k=1}^{L} a_{k} E_{c u}^{k}+a_{L+1} E_{c u}^{L+1}}{b_{0}+\sum_{k=1}^{L} b_{k} E_{c u}^{k}+b_{L+1} E_{c u}^{L+1}},
$$


with constraints of $\sum_{k=1}^{L+1} E_{c u}^{k}=E_{\mathrm{CUs}}$ and $E_{c u}^{k} \geq 0$. We Apply (31) for $K=L$ to (35) and obtain

$$
\begin{aligned}
& \max _{\sum_{k=1}^{L+1} E_{c u}^{k}=E_{\mathrm{CUs}}} \frac{a_{0}+\sum_{k=1}^{K+1} a_{k} E_{c u}^{k}}{b_{0}+\sum_{k=1}^{K+1} b_{k} E_{c u}^{k}} \\
& =\max _{0 \leq E_{c u}^{L+1} \leq E_{\mathrm{CUs}} \sum_{k=1}^{L} E_{c u}^{k}=E_{\mathrm{CUs}}-E_{c u}^{L+1}} \frac{a_{0}+\sum_{k=1}^{L} a_{k} E_{c u}^{k}+a_{L+1} E_{c u}^{L+1}}{b_{0}+\sum_{k=1}^{L} b_{k} E_{c u}^{k}+b_{L+1} E_{c u}^{L+1}} \\
& =\max _{0 \leq E_{c u}^{L+1} \leq E_{\mathrm{CUs}}} \max _{k=1, \ldots, L} \frac{a_{0}+a_{k}\left(E_{\mathrm{CUs}}-E_{c u}^{L+1}\right)+a_{L+1} E_{c u}^{L+1}}{b_{0}+b_{k}\left(E_{\mathrm{CUs}}-E_{c u}^{L+1}\right)+b_{L+1} E_{c u}^{L+1}} .
\end{aligned}
$$

We switch the maximum operations on the right-hand side to have

$$
\begin{gathered}
\max _{\sum_{k=1}^{L+1} E_{c u}^{k}=E_{\mathrm{CUs}}} \frac{a_{0}+\sum_{k=1}^{K+1} a_{k} E_{c u}^{k}}{b_{0}+\sum_{k=1}^{K+1} b_{k} E_{c u}^{k}} \\
=\max _{k=1, \ldots, L} \max _{0 \leq E_{c u}^{L+1} \leq E_{\mathrm{CUs}}} \frac{a_{0}+a_{k}\left(E_{\mathrm{CUs}}-E_{c u}^{L+1}\right)+a_{L+1} E_{c u}^{L+1}}{b_{0}+b_{k}\left(E_{\mathrm{CUs}}-E_{c u}^{L+1}\right)+b_{L+1} E_{c u}^{L+1}} .
\end{gathered}
$$

Due to the monotonicity of (32), we can easily get

$$
\begin{aligned}
& \max _{\sum_{k=1}^{L+1} E_{c u}^{k}=E_{\mathrm{CUs}}} \frac{a_{0}+\sum_{k=1}^{K+1} a_{k} E_{c u}^{k}}{b_{0}+\sum_{k=1}^{K+1} b_{k} E_{c u}^{k}} \\
= & \max _{k=1, \ldots, L} \max \left(\frac{a_{0}+a_{k} E_{\mathrm{CUs}}}{b_{0}+b_{k} E_{\mathrm{CUs}}}, \frac{a_{0}+a_{L+1} E_{\mathrm{CUs}}}{b_{0}+b_{L+1} E_{\mathrm{CUs}}}\right) \\
= & \max _{k=1, \ldots, L+1} \frac{a_{0}+a_{k} E_{\mathrm{CUs}}}{b_{0}+b_{k} E_{\mathrm{CUs}}} .
\end{aligned}
$$

Therefore, (31) also holds for $K=L+1$, which proves (20a) by mathematical induction.

\section{Abbreviations}

NSCRS: Non-selfish symbiotic cognitive relaying scheme; PU: Primary user; CUs: Cognitive users; CSI: Channel state information; WSN: Wireless sensor network; EE: Energy efficiency; AF: Amplify-and-forward; DF: Decoded-and-forward; NAF: Non-orthogonal AF; AP: Access point; CCR: Conventional cognitive radio scheme; TDMA: Time division multiple address; AN: Artificial noise; SNR: Signal-to-noise ratio; AWGN: Additive white Gaussian noise; EPA: Equal energy allocation algorithm; OPA: Optimal energy and time allocation algorithm with full CSI; PPA: Partial CSI feedback based suboptimal energy and time allocation algorithm.

\section{Acknowledgements}

The authors thank the person who provided meticulous and valuable suggestions for improving the paper.

\section{Authors' contributions}

PL and PG proposed the overall research direction and ideas. LH and PG designed the system model and the resource allocation algorithm. LH and XG drafted the article and designed and the simulations. HL read the relevant literature and revised the manuscript. The authors read and approved the final manuscript.

\section{Funding}

This work was supported in part by the National Natural Science foundation of China under Grant 62073039 and National Key Research and Development Program of China under Grant 2018 YFC0823003.

\section{Data availibility}

Data sharing is not applicable to this article as no datasets are generated or analyzed during the current study.

\section{Competing interests}

The authors declare that they have no competing interests.

Received: 31 July 2020 Accepted: 15 January 2021

Published online: 08 March 2021 
References

1. L. Bai, G. Li, H. Zhu, Privacy protection algorithm based on linear directional phantom source node in WSN, in: 2017 8th IEEE International Conference on Software Engineering and Service Science (ICSESS), Beijing, (2017)pp. 851-854

2. P.K. Roy, J.P. Rimjhim, Singh, P. Kumar, An efficient privacy preserving protocol for source location privacy in wireless sensor networks, in: 2016 International Conference on Wireless Communications, Signal Processing and Networking (WiSPNET), Chennai (2016), pp. 1093-1097

3. T. Rault, A. Bouabdallah, Y. Challal, Energy efficiency in wireless sensor networks: a top-down survey. Comput. Netw. 67(4), 104-122 (2014)

4. M. Bagaa, A. Chelli, D. Djenouri, T. Taleb, I. Balasingham, K. Kansanen, Optimal placement of relay nodes over limited positions in wireless sensor networks. IEEE Trans. Wirel. Commun. 16(4), 2205-2219 (2017)

5. Y. Zhao, R. Adve, T.J. Lim, Improving amplify-and-forward relay networks: optimal power allocation versus selection. IEEE Trans. Wirel. Commun. 6(8), 3114-3123 (2007)

6. K.T. Phan, T. Le-Ngoc, S.A. Vorobyov, C. Tellambura, Power allocation in wireless multi-user relay networks. IEEE Trans. Wirel. Commun. 8(5), 2535-2545 (2009)

7. P. Gong, P. Xue, D. Park, D.K. Kim, Optimum power allocation in a nonorthogonal amplify-and-forward relay-assisted network. IEEE Trans. Veh. Technol. 60(3), 890-900 (2011)

8. M. Elsaadany, W. Hamouda, Performance analysis of non-orthogonal AF relaying in cognitive radio networks. IEEE Wirel. Commun. Lett. 4(4), 373-376 (2015)

9. Y. Jing, H. Jafarkhani, Single and multiple relay selection schemes and their achievable diversity orders. IEEE Trans. Wirel. Commun. 8(3), 1414-1423 (2009)

10. I. Kim, D. Kim, Minimizing operational power cost in remote-area wireless sensor networks with a DF relay and outage constraints. IEEE Commun. Lett. 19(2), 247-250 (2015)

11. K. Hamdi, M.O. Hasna, A. Ghrayeb, K.B. Letaief, Opportunistic spectrum sharing in relay-assisted cognitive systems with imperfect CSI. IEEE Trans. Veh. Technol. 63(5), 2224-2235 (2014)

12. N. Tuah, M. Ismail, Extending lifetime of heterogenous wireless sensor network using relay node selection, in: 2013 International Conference of Information and Communication Technology (ICo/CT), Bandung (2013), pp. 17-21

13. M. Saber, M. Naznin, Saving relay time in a heterogeneous wireless sensor network, in: 2018 IEEE International Symposium on Local and Metropolitan Area Networks (LANMAN), Washington (2018), pp. 122-124

14. S. Sapre, S. Mini, Relay node placement to establish full connectivity in directional sensor networks, in: 2017 Fourteenth International Conference on Wireless and Optical Communications Networks (WOCN), Mumbai (2017), pp. 1-5

15. C. Ma, W. Liang, M. Zheng, H. Sharif, A connectivity-aware approximation algorithm for relay node placement in wireless sensor networks. IEEE Sens. J. 16(2), 515-528 (2016)

16. K. Azarian, H. El Gamal, P. Schniter, On the achievable diversity-multiplexing tradeoff in half-duplex cooperative channels. IEEE Trans. Inf. Theory 51(12), 4152-4172 (2005)

17. L.J. Rodriguez, N.H. Tran, T. Le-Ngoc, On the capacity of the static half-duplex non-orthogonal AF relay channel. IEEE Trans. Wirel. Commun. 13(2), 1034-1046 (2014)

18. M. Elsaadany, Optimal power allocation in cognitive networks using non-orthogonal AF relays, in: 39th Annual IEEE Conference on Local Computer Networks, Edmonton (2014), pp. 410-413

19. L. Guo, B. Wang, W. Wang, Research of energy-efficiency algorithm based on on-demand load balancing for wireless sensor networks, in: 2009 International Conference on Test and Measurement, Hong Kong, vol. 2 (2009), pp. 22-26

20. H. Xu, L. Sun, P. Ren, Q. Du, Y. Wang, Cooperative privacy preserving scheme for downlink transmission in multiuser relay networks. IEEE Trans. Inf. Forensics Secur. 12(4), 825-839 (2017)

21. S. Haykin, Cognitive radio: brain-empowered wireless communications. IEEE J. Sel. Areas Commun. 23(2), 201-220 (2005)

22. N. ul Hasan, W. Ejaz, M.K. Atiq, H.S. Kim, Distributed connectivity restoration using cognitive relay nodes positioning in damaged wireless sensor network, in: 2013 IEEE Symposium on Wireless Technology Applications (ISWTA), Kuching (2013), pp. 159-162

23. K. Tang, R. Shi, J. Dong, Throughput analysis of cognitive wireless acoustic sensor networks with energy harvesting. Future Gener Comput Syst 86, 1218-1227 (2017)

24. B. Han, M. Zeng, Q. Guo, H. Jiang, Q. Zhang, L. Feng, Energy-efficient sensing and transmission for multi-hop relay cognitive radio sensor networks. China Commun 15(9), 106-117 (2018)

25. P. Gong, J.H. Park, J.M. Yoo, B. Yu, D.K. Kim, Throughput maximization with multiuser non-selfish cognitive relaying in CR networks, in: 2009 4th International Symposium on Wireless Pervasive Computing, Melbourne (2009), pp. 1-5

26. O. Simeone, I. Stanojev, S. Savazzi, Y. Bar-Ness, U. Spagnolini, R. Pickholtz, Spectrum leasing to cooperating secondary ad hoc networks. IEEE J. Sel. Areas Commun. 26(1), 203-213 (2008)

27. A. Mercado, B. Azimi-Sadjadi, Power efficient link for multi-hop wireless network, in: Proceedings of the 41st Allerton Conference on Communication, Control, and Computing, Monticello, USA (2003), pp. 1837-1847

\section{Publisher's Note}

Springer Nature remains neutral with regard to jurisdictional claims in published maps and institutional affiliations. 